\title{
Experiential Effects of Appetitive and Nonappetitive Odors on Feeding Behavior in the Blowfly, Phormia regina: A Putative Role for Tyramine in Appetite Regulation
}

\author{
Tomoyosi Nisimura, ${ }^{1 \star}$ Atsushi Seto, ${ }^{1 *}$ Kyoko Nakamura, ${ }^{1}$ Mayumi Miyama, ${ }^{1}$ Takashi Nagao, ${ }^{2}$ Satoshi Tamotsu, ${ }^{3}$ \\ Ryohei Yamaoka, ${ }^{1}$ and Mamiko Ozaki ${ }^{1}$ \\ ${ }^{1}$ Department of Applied Biology, Faculty of Textile Science, Kyoto Institute of Technology, Kyoto 606-8585, Japan, ${ }^{2}$ Human Information Systems, Kanazawa \\ Institute of Technology, Ishikawa 924-0838, Japan, and ${ }^{3}$ Graduate School of Human Culture, Nara Women’s University, Nara 630-8506, Japan
}

\begin{abstract}
In humans, appetite is affected by food experiences and food flavors. In the blowfly Phormia regina, we found that feeding threshold to sugar increased in the presence of the odor of D-limonene and decreased in the presence of the odor of dithiothreitol (DTT). Using these odors as representative nonappetitive and appetitive flavors, we demonstrated the role played by tyramine (TA) in appetite regulation by experiences of food flavors. When fed with sucrose flavored with $\mathrm{D}$-limonene for $5 \mathrm{~d}$ after emergence, flies showed subsequent decreased appetite to plain sucrose, whereas when they were fed with sucrose flavored by DTT they showed increased appetite. However, mushroom body (MB)-ablated flies did not show these patterns. This suggests that MB, one of the primary memory centers of the insect brain, is necessary for the flies to apply previous experiences of food flavors to appetitive learning behaviors. In addition, flies' previously acquired decreased or increased appetites showed parallel changes with both octopamine $(\mathrm{OA})$ and tyraminelevels in the brain. However, injection experiments with $\mathrm{OA}, \mathrm{TA}$, or their agonist and antagonist indicated that TA more directly mediates feeding threshold determination, which was affected by acquired memories of food flavors.
\end{abstract}

Key words: appetite; food flavor; experience; tyramine; mushroom body; learning and memory

\section{Introduction}

It is known that food preference in humans is affected by individual dietary experiences, familial traditional tastes, or human food cultures. Higher animal species, including humans, are expected to learn from experiences to determine their food preference. This is also true for insects.

In flies, and some other insects, the proboscis extension reflex (PER) has long been used as an indicator of behavioral sensitivity for taste studies (Dethier, 1976; Devaud, 2003; Ozaki et al., 2003; Ishimoto and Tanimura, 2004; Scheiner et al., 2004). Flies extend their proboscis when contact chemosensilla on their legs or labela detect sugar above a certain threshold concentration. Thus, we used PER as a measure of appetite.

The PER threshold is affected by experiences or previous learning. In Phormia regina, PER threshold depends on daily food concentration of sugar (Yano et al., 1986), and, in Apis mellifera, it depends on foraging experiences and/or age (Pankiw and Page, 1999; Pankiw et al., 2001; Page and Erber, 2002). Moreover, PER

Received Feb. 4, 2005; revised June 21, 2005; accepted June 21, 2005

This work was supported by a grant from Human Frontier Science Program and the Program for Promotion of Basic Research Activities for Innovative Biosciences (M.O.). We thank Dr. R. Raguso (University of Michigan, Ann Arbor, MI) for critical reading of this manuscript.

*T.N. and A.S. contributed equally to this work.

Correspondence should be addressed to Dr. Mamiko Ozaki, Department of Applied Biology, Faculty of Textile Science, Kyoto Institute of Technology, Kyoto 606-8585, Japan. E-mail: mamiko@ipc.kit.ac.jp.

D0I:10.1523/JNEUROSCI.1862-05.2005

Copyright $\odot 2005$ Society for Neuroscience $\quad$ 0270-6474/05/257507-10\$15.00/0 can be conditioned across sensory modalities, to salt stimulation by saltiness-sweetness-associative learning in P. regina (Akahane and Amakawa, 1983), and can be inhibited by sweetnessbitterness-associative learning in Drosophila melanogaster (Médioni and Vaysse, 1975; DeJianne et al., 1985; Brigui et al., 1990). However, in the flies $P$. regina and D. melanogaster, classical conditioning for taste-olfaction-associative learning using PER has not been satisfactorily demonstrated.

Previously, odorants were classified into attractants or repellents with the T-maze binary choice assays using D. melanogaster (Ayyub et al., 1990; Devaud, 2003). Attractants and repellents are not necessarily functionally equivalent to appetitive and nonappetitive odors, but flies may effectively identify potentially toxic chemicals by their odors. If such odors were reliable indicators of toxicity, natural selection could result in their perception as fixed nonappetitive odors, which would decrease appetite in flies. Recently, Ozaki et al. (2003) found that taste stimulation of the deterrent taste cell with D-limonene, a volatile monoterpene, induced strong aversive behavior in $P$. regina. Thus, vapor of D-limonene could act as an olfactory cue to prevent flies from feeding.

At the neural level, feeding behavior is known to be regulated by biogenic amines that play various important roles in behavior regulation in insect brains (Evans, 1980; Roeder, 1994; Roeder et al., 2003). In the honeybee, octopamine (OA) and its agonists were shown to enhance elicitation of PER (Erber et al., 1993; Blenau and Erber, 1998) and to increase PER sensitivity to su- 
crose (Scheiner et al., 2002). In addition, in P. regina, OA, dopamine (DA), and serotonin are involved in PER sensitivity to sucrose (Long and Murdock, 1983; Long et al., 1986; Brookhart et al., 1987), suggesting that OA is involved in appetite in $P$. regina. In this study, we also used $P$. regina as an animal model and studied the underlying mechanism of appetite regulation by experiences with different food flavors.

\section{Materials and Methods}

Flies. The blowfly Phormia regina M. was originally donated from Prof. H. Morita's laboratory in Kyushu University (Fukuoka, Japan) and reared in our laboratory under a $12 \mathrm{~h}$ light/dark cycle at $22 \pm 2^{\circ} \mathrm{C}$. Larvae were fed with chicken liver and yeast bait (Oriental Yeast, Tokyo, Japan) unless otherwise stated. Adults were provided with water and $0.1 \mathrm{M} \mathrm{su-}$ crose solution in separate cups.

To collect eggs, flies older than $7 \mathrm{~d}$ after emergence were reared in a separate cage, being provided with water and sucrose plus chicken liver. Egg masses laid on chicken livers were collected every morning.

Experimental paradigm for dietary experience with odors. An adult fly population derived from the same egg mass was divided into two groups within $1 \mathrm{~d}$ after emergence and reared in separate plastic cages $(22 \times 15 \times$ $13 \mathrm{~cm}^{3}$ ) under different dietary conditions. One group (experienced group) of flies was provided with water in a cup and $0.1 \mathrm{~m}$ sucrose solution flavored with D-limonene or dithiothreitol (DTT) on a special meal stage for $5 \mathrm{~d}$. In a double-bottomed plastic meal stage $(75 \mathrm{~mm}$ diameter; $35 \mathrm{~mm}$ height), a cotton ball soaked with sucrose solution was placed on the upper dish, and pure D-limonene oil or DTT powder was placed in the lower compartment, which was mostly airtight except for pinhole openings at the edge of the upper dish. When flies visited the meal stage and extended their proboscis to feed on sucrose, they would be stimulated with the taste of sugar and the odor of D-limonene or DTT at the same time. As a control group (nonexperienced group), the other flies were provided with water and $0.1 \mathrm{~m}$ sucrose on the same meal stage, in which no odor source was present for $5 \mathrm{~d}$. For the following $2 \mathrm{~d}$, both groups of flies were provided only with water until behavioral testing. Temperature was maintained at $22 \pm 2^{\circ} \mathrm{C}$.

PER test for appetite measurement. In previous studies, appetite has often been measured by the amount of food intake and/or excretion. However, in the present paper, the term "appetite" was used to indicate the motivation for feeding, which we evaluated by using the threshold for PER, which is a necessary prerequisite to feeding. Decreased and increased appetites in flies would indicate high and low PER thresholds to sucrose, respectively. With the PER test, we obtained thresholds for individual flies and compared them between populations. Twenty to 40 individuals were randomly chosen from each group and were immobilized by securing the wings with aluminum clothes pegs. Before the PER test, flies were provided with distilled water to satiation. Stimulus solutions for the PER test were 11 steps of sucrose concentrations, which were prepared by twofold serial dilutions with distilled water starting from a sucrose concentration of $1 \mathrm{M}$. Labelar chemosensilla of flies were carefully stimulated with each of the sucrose concentrations beginning with the lowest concentration, so that flies would not ingest stimulus solutions. When we examined the effects of the presence of odors, the PER test was performed with the odor source set $\sim 2 \mathrm{~cm}$ away from the fly. The sucrose concentration to which the fly first extended its proboscis was defined as the PER threshold at the individual level. Because PER thresholds of individuals from a group were normally distributed according to the logarithmic scale of sucrose concentration, we calculated the mean of PER thresholds at the population level with the probit analysis computer program of Prof. M. Sakuma (Kyoto University, Kyoto, Japan) (Sakuma, 1998). If we obtained data for at least four concentration-PER curves per condition (see Figs. 1,2), statistical differences in the means of PER thresholds between groups, which were reared and/or examined under different conditions, were tested by Tukey's test. When we obtained data for less than four concentration-PER curves per condition (see Figs. 3, 4, $8,9)$, statistical differences were tested not within the means of PER thresholds but for individual PER threshold. We performed at least duplicate PER tests per condition. Inconveniently, concentration-PER curves obtained even under the same experimental conditions indicated more or less different population variances. Thus, to test statistical differences in individual PER thresholds of duplicated data between groups, we used a nonparametric Steel-Dwass test rather than a parametric Tukey's test.

Ablation of mushroom bodies. We ablated mushroom bodies (MBs) of the blowfly following the methods of de Belle and Heisenberg (1994) in Drosophila and of Ikeda et al. (2005) in the blow fly Protophormia terraenovae. The newly hatched larvae from the same egg lump were divided into two groups. One group of control larvae followed a normal artificial diet $[7 \%$ dried yeast, $7 \%$ wheat germ, $7 \%$ whole milk powder, $1.4 \%$ agar, $0.12 \%$ brilliant blue FCF (w/v), and $0.5 \%$ propionic acid (v/v) (Tachibana and Numata, 2001)] for $4 \mathrm{~h}$. The other group of larvae was fed with hydroxyurea (HU)-plus diet (basic diet plus $25 \mathrm{mg} / \mathrm{ml} \mathrm{HU}$ ) for $4 \mathrm{~h}$. The larvae whose guts were stained blue were selectively transferred to their regular diet of chicken liver and yeast bait. When they reached adulthood, flies were subjected to PER tests. Subsequently, they were histologically examined to verify whether MBs had been ablated in the brain.

Histological examination of the brain. Flies' heads were isolated on ice, and the proboscis and the posterior cuticle were removed and placed in PBS. They were then fixed with Bouin's fluid (saturated aqueous picric acid/formaldehyde/glacial acetic acid at 15:5:1) and kept overnight at room temperature. Brains were dehydrated and embedded in Parablast (Nakarai Tesque, Kyoto, Japan). Serial thin sections $(7 \mu \mathrm{m})$ of brains were made with a rotary microtome (RV-240; Yamato Kohki Industrial, Saitama, Japan), stained with Mayer's acid hematoxylin and eosin, and observed under a photomicroscope (ECLIPSE E200; Nikon Instech, Tokyo, Japan). We examined every section to check for the existence of calyces, peduncles, and/or lobes of MBs.

Sample preparation for biogenic amine measurement. Brains for biogenic amine measurements were sampled from individuals with the nearest PER threshold to the mean PER threshold of that particular population. Heads of the sample flies in each test or control group were stored in an Eppendorf tube at $-80^{\circ} \mathrm{C}$. Heads were lyophilized overnight at $-50^{\circ} \mathrm{C},<0.1$ torr by a freeze-dry system (FZ-4.5; Labconco, Kansas City, MO). Brains were isolated from lyophilized heads, and optic lobes were removed. They were then homogenized on ice in a microglass homogenizer with $50 \mu \mathrm{l}$ of chilled $0.1 \mathrm{M}$ perchloric acid containing the internal standard 3, 4-dihydroxybenzylamine at $25 \mathrm{ng} / \mathrm{ml}$. Each homogenate was transferred into an Eppendorf tube and centrifuged at 15,000 rpm for $30 \mathrm{~min}$ at $0^{\circ} \mathrm{C}$. The supernatant was applied to a microvial for HPLC-electrochemical (ECD) analysis.

HPLC-ECD analysis. For simultaneous determination of biogenic amines, we used a modified method of the original procedure by Nagao and Tanimura $(1988,1989)$. The HPLC system was equipped with a solvent delivery pump (model 515; Waters Associates, Milford, MA), a refrigerated automatic injector (model 231-401; Gilson, Middleton, WI), and $\mathrm{a} \mathrm{C}_{18}$ reversed-phase column $(250 \times 4.6 \mathrm{~mm}$ inner diameter, $5 \mu \mathrm{m}$ average particle size; model UG120; Shiseido, Tokyo, Japan) maintained at $30^{\circ} \mathrm{C}$ in a column oven. The detector cell was also kept at $30^{\circ} \mathrm{C}$ in the column oven. An electrochemical detector with a glassy carbon electrode (WE-GC; Eicom, Kyoto, Japan) was used. Detector potential was set at $0.85 \mathrm{~V}$ against an $\mathrm{Ag} / \mathrm{AgCl}$ reference electrode. Signals from the detector were recorded and integrated by using data analysis software (Millennium; Waters Associates).

The mobile phase for HPLC consisted of a solution containing $0.18 \mathrm{M}$ monochloroacetic acid and $40 \mu \mathrm{m} 2 \mathrm{Na}$-EDTA, adjusted to $\mathrm{pH} 3.6$ with $\mathrm{NaOH}$, and combined with $1.62 \mathrm{~mm}$ sodium-1-octanesulfonate. This solution was then filtrated through a $0.22 \mu \mathrm{m}$ filter (Millipore, Billerica, MA), degassed with a vacuum pump, and kept at a constant flow of $0.7 \mathrm{ml} / \mathrm{min}$. The external standard mixture was made up of 27 different compounds; 3,4-dihydroxy mandelic acid; $\beta$-3,4-dihydroxy o-phenylalanine; 4-hydroxy3-methoxymandelic acid; tyrosine; 4-hydroxy-3-methoxy phenylglycol; $\mathrm{N}$ acetyloctopamine; norepinephrine; 5-hydroxy-L-tryptophane; epinephrine; OA; 3,4-dihydroxybenzylamine; 3,4-dihydroxyphenylacetic acid; normetanephrine; synephrine; $N$-acetyldopamine (NADA); DA; metanephrine; deoxyepinephrine; 5-hydroxyindole-3-acetic acid; $N$-acetyltyramine (NATA); tyramine (TA); $N$-acetyl-5-hydroxytryptamine; 3-methoxy-4-hydroxy phenyl acetic acid; tryptophan; 3-methoxytyramine; 5-hydroxytryptamine (5- 


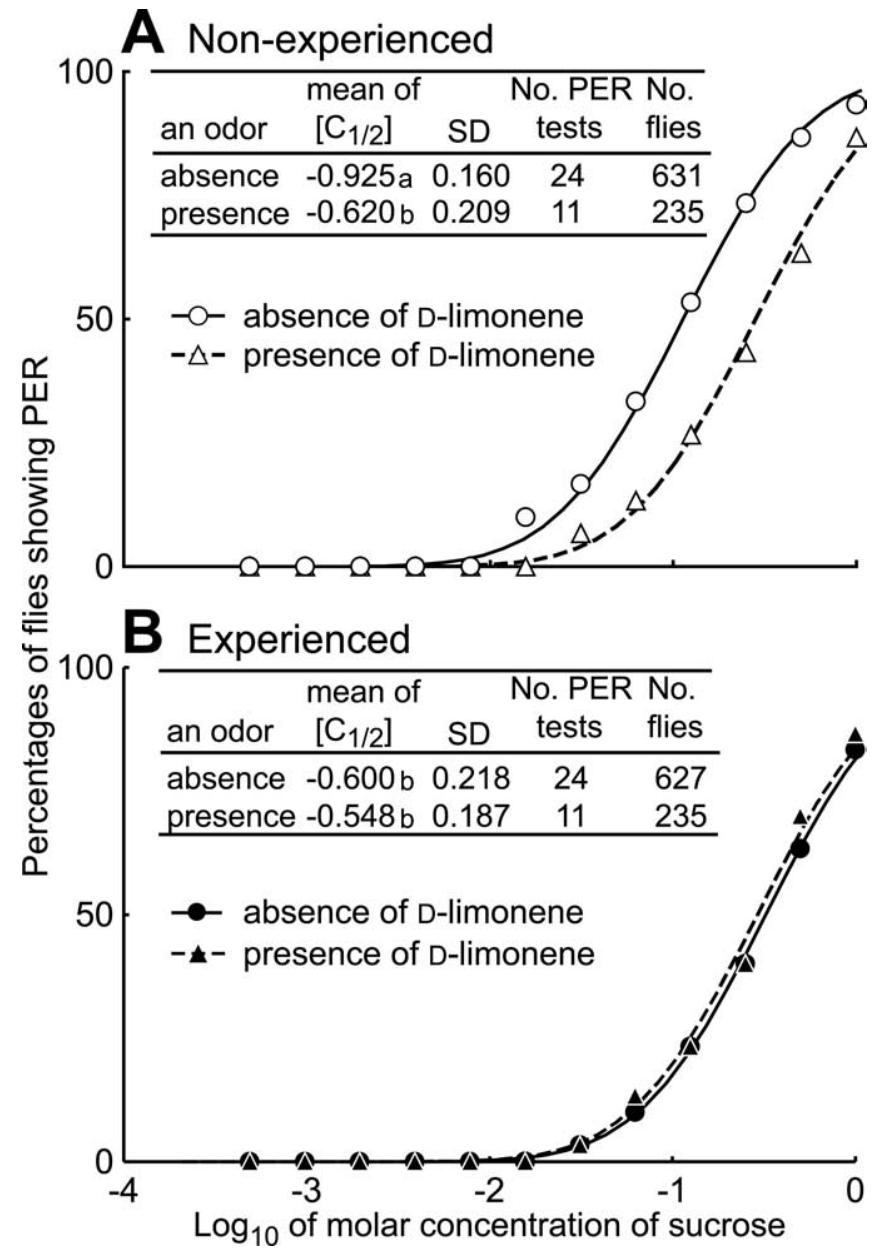

Figure 1. Effects of the odor of D-limonene on PER threshold. Flies were fed with $0.1 \mathrm{M}$ sucrose without (open symbols; $\boldsymbol{A}$ ) or with (filled symbols; $\boldsymbol{B}$ ) the odor of D-limonene for $5 \mathrm{~d}$ after emergence. The PER test was performed $7 \mathrm{~d}$ after emergence in the absence (circles) or the presence (triangles) of the odor. Sigmoidal curves with circles or triangles show representative results of the PER tests in a fly group. Figures $1-4,8$, and 9 show similar inset tables, in which $\left[C_{1 / 2}\right]$ indicates PER threshold at the population level (for details, see Materials and Methods). Means of $\left[C_{1 / 2}\right]$ with the same alphabetical letters have no significant differences with each other ( $p>0.05$, Tukey's test).

HT); and 6-hydroxymelatonine. This external standard mixture was run three times per HPLC operation: before and after test sample applications (12-16 samples were applied at once) and between test sample applications. Referring to the elution pattern of the external standard mixture and the peak area of 3,4-dihydroxybenzylamine commonly used as internal and external standards, peaks for OA, NADA, DA, NATA, TA, and 5-HT in the test samples were identified, and their amounts were calculated.

Reagent injection to flies. Appropriate concentrations of clonidine hydrochloride, phentolamine, OA, or TA were dissolved in Ringer's solution (in mM: $111.2 \mathrm{NaCl}, 5.5 \mathrm{KCl}, 0.08 \mathrm{NaH}_{2} \mathrm{PO}_{4} \cdot 2 \mathrm{H}_{2} \mathrm{O}, 1.2 \mathrm{NaHCO}_{3}, 1.8$ $\mathrm{CaCl}_{2} \cdot 2 \mathrm{H}_{2} \mathrm{O}, 0.8 \mathrm{MgCl}_{2} \cdot 6 \mathrm{HO}$, and 5 HEPES-Na). Approximately $45 \mathrm{~min}$ before the beginning of the PER test, $1 \mu \mathrm{l}$ of solution of one of these reagents was injected into the thorax of the flies in the test group, and the same volume of Ringer's solution was injected into the thorax of the control group. To compare the effects between reagents or doses, the mean of PER thresholds in a reagent-injected group, $\left[\mathrm{C}_{1 / 2}\right]_{\mathrm{i}}$, was normalized to the mean of PER thresholds in the nonexperienced Ringer's solution-injected group, $\left[\mathrm{C}_{1 / 2}\right]_{\mathrm{o}}$, so that the mean of PER thresholds in the reagent-injected group was relatively evaluated by $\left[\mathrm{C}_{1 / 2}\right]_{\mathrm{i}} /\left[\mathrm{C}_{1 / 2}\right]_{\mathrm{o}}$. When expressed in a logarithmic scale, $\log _{10}\left(\left[\mathrm{C}_{1 / 2}\right]_{\mathrm{i}} /\left[\mathrm{C}_{1 / 2}\right]_{\mathrm{o}}\right)=$ $\log _{10}\left[\mathrm{C}_{1 / 2}\right]_{\mathrm{i}}-\log _{10}\left[\mathrm{C}_{1 / 2}\right]_{\mathrm{o}}$. If $\log _{10}\left[\mathrm{C}_{1 / 2}\right]_{\mathrm{i}}-\log _{10}\left[\mathrm{C}_{1 / 2}\right]_{\mathrm{o}}<0$, the injected reagent elicited an increase of appetite, and if $\log _{10}\left[\mathrm{C}_{1 / 2}\right]_{\mathrm{i}}-$ $\log _{10}\left[\mathrm{C}_{1 / 2}\right]_{\mathrm{o}}>0$, the injected reagent elicited a decrease in appetite.

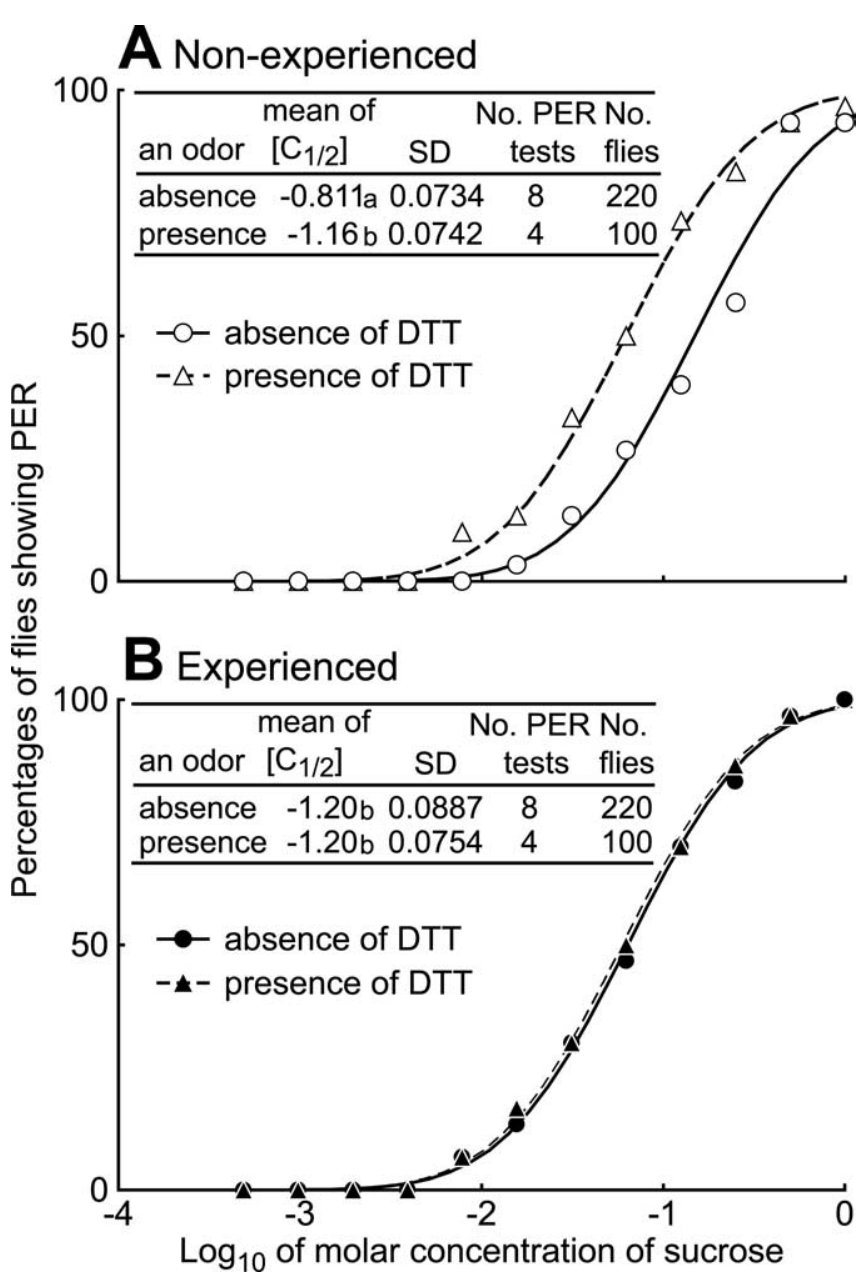

Figure 2. Effects of the odor of DTT on PER threshold to sucrose. Flies were fed with $0.1 \mathrm{M}$ sucrose without (open symbols; $\boldsymbol{A}$ ) or with (filled symbols; $\boldsymbol{B}$ ) the odor of DIT for $5 \mathrm{~d}$ after emergence. The PER test was performed $7 \mathrm{~d}$ after emergence in the absence (circles) or the presence (triangles) of the odor. Sigmoidal curves with circles or triangles show representative results of the PER tests in a fly group. Means of $\left[C_{1 / 2}\right]$ with the same alphabetical letters have no significant differences with each other ( $p>0.05$, Tukey's test).

\section{Results}

Effects of the presence and dietary experience of the odor of D-limonene on PER threshold to sucrose in intact flies

In the nonexperienced groups (Fig. $1 A$ ), using different batches of flies, we repeated the same PER test 24 and 11 times in the absence and the presence of the odor of D-limonene, respectively. The average value of $\log _{10}\left[\mathrm{C}_{1 / 2}\right] \pm \mathrm{SD}$ was then calculated to be $-0.925 \pm 0.160$ when tested in the absence of the odor of D-limonene or was $-0.620 \pm 0.209$ when tested in the presence of the odor of D-limonene. These values were significantly different from each other $(p<0.05$, Tukey's test). In addition, in the experienced groups (Fig. $1 B$ ), we performed the PER test in the absence or the presence of the odor of D-limonene and drew concentration-PER curves. In these experienced groups, the average value of $\log _{10}\left[C_{1 / 2}\right] \pm S D$ was $-0.600 \pm 0.218$ in the absence of the odor of D-limonene $(n=24)$ and $-0.548 \pm 0.187$ in the presence of the odor of D-limonene $(n=11)$. There was no significant difference between these values $(p>0.05$, Tukey's test).

The $\log _{10}\left[\mathrm{C}_{1 / 2}\right]$ value obtained by the PER test in the absence of the odor of $\mathrm{D}$-limonene in the experienced group, i.e., $-0.600 \pm 0.218(n=24)$ (Fig. $1 B)$, was significantly higher than 
that in the nonexperienced group, which was $-0.925 \pm 0.160(n=24)$ (Fig. $1 A)$ ( $p<0.05$, Tukey's test). Thus, dietary experience with the odor of D-limonene decreased appetite to plain sucrose, mimicking the presence of olfactory stimulus with $\mathrm{D}$-limonene. Indeed, $\left[\mathrm{C}_{1 / 2}\right]$ values for the experienced groups obtained in the absence of the odor of $\mathrm{D}$-limonene with $\log _{10}\left[\mathrm{C}_{1 / 2}\right]$ of $-0.600 \pm 0.218(n=24)$ (Fig. $1 B$ ) were mostly the same as $\left[\mathrm{C}_{1 / 2}\right]$ values for the nonexperienced groups in the presence of the odor of D-limonene in which $\log _{10}\left[\mathrm{C}_{1 / 2}\right]$ was $-0.620 \pm 0.209$ $(n=11)$ (Fig. $1 A)$. There was no significant difference between these values $(p>$ 0.05 , Tukey's test).

\section{Effects of the presence and dietary experience of the odor of DTT on PER threshold to sucrose in intact flies} Using nonexperienced groups, in which flies were fed with plain sucrose for $5 \mathrm{~d}$ after emergence, and experienced groups, in which flies were fed with sucrose flavored by the odor of DTT, we performed the PER test in the absence or the presence of the odor of DTT and determined $\left[\mathrm{C}_{1 / 2}\right]$ (Fig. 2). In the nonexperienced groups (Fig. 2A), $\log _{10}\left[\mathrm{C}_{1 / 2}\right]$ was $-0.811 \pm$ 0.0734 when tested in the absence of the odor of DTT $(n=8)$ and $-1.16 \pm 0.0742$ when tested in the presence of the odor of DTT $(n=4)$. These values were significantly different from each other $(p<0.05$, Tukey's test). Also, in the experienced groups (Fig. $2 B$ ), we performed the PER test in the absence or the presence of the odor of DTT. It was then calculated that $\log _{10}\left[\mathrm{C}_{1 / 2}\right]$ was $-1.20 \pm 0.0887$ in the absence of the odor of DTT $(n=8)$ and $-1.20 \pm 0.0754$ in the presence of the odor of DTT $(n=4)$. There was no significant difference between these values $(p>$ 0.05, Tukey's test).

The values of $\log _{10}\left[\mathrm{C}_{1 / 2}\right]$ obtained by the PER test in the absence of the odor of DTT were $-0.811 \pm 0.0734$ in the nonexperienced groups $(n=8)$ (Fig. $2 A)$ and $-1.20 \pm 0.0887$ in the experienced groups $(n=8)$ (Fig. $2 B)$. These values were significantly different from each other ( $p<0.05$, Tukey's test). $\log _{10}\left[\mathrm{C}_{1 / 2}\right]$ values of the experienced groups obtained in the absence of the odor of DTT, i.e., $-1.20 \pm 0.0887(n=8)$ (Fig. $2 B$ ), were mostly the same as $\log _{10}\left[\mathrm{C}_{1 / 2}\right]$ values of the nonexperienced groups in the presence of the odor of DTT, i.e., $-1.16 \pm 0.0742$ $(n=4)$ (Fig. $2 A)(p>0.05$, Tukey's test). Thus, dietary experience with the odor of DTT increased appetite to plain sucrose, mimicking the presence of olfactory stimulus with DTT.

\section{Effects of the presence and dietary experience of the odor of D-limonene on PER threshold to sucrose in MB-ablated flies}

In the nonexperienced group of MB-ablated flies made by HUtreatment, PER threshold to sucrose was increased in the presence of the odor of D-limonene (Fig. 3A). In PER duplicate tests, $\log _{10}\left[C_{1 / 2}\right]$ was -0.79 in the absence of the odor and -0.33 in the presence of the odor (data from representative PER curve shown in Fig. $3 A$ ) for one data set, and $\log _{10}\left[C_{1 / 2}\right]$ of -1.39 in the ab-
HU-treated

\section{Control}

Non-experienced

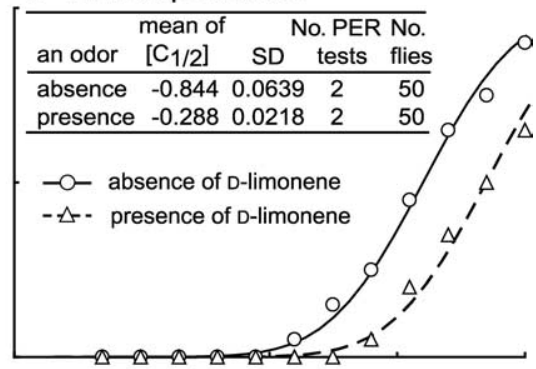

D Experienced

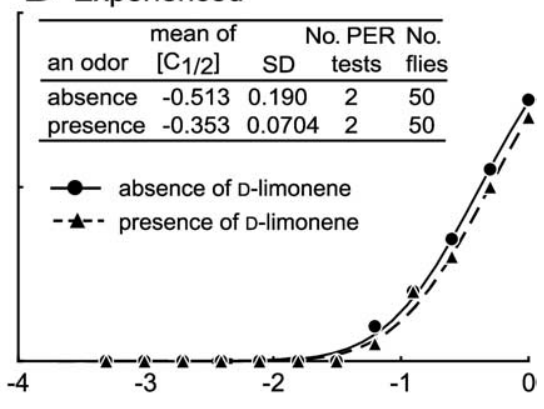

Log10 of molar concentration of sucrose sence of the odor and -0.56 in the presence of the odor for another data set. Differences in individual PER thresholds between presence and absence of the odor were statistically significant ( $p<0.05$, Steel-Dwass test; $n=50)$. This was similar to the nonexperienced intact fly group (Fig. 3C). In PER duplicate tests, $\log _{10}\left[\mathrm{C}_{1 / 2}\right]$ was -0.80 in the absence of the odor and -0.30 in the presence of the odor (data from the curve in Fig. $3 C$ ) for one data set, and $\log _{10}\left[\mathrm{C}_{1 / 2}\right]$ was -0.89 in the absence of the odor and -0.27 in the presence of the odor for another data set. These results indicated that, even without $\mathrm{MBs}$, the fly appetite decreased in the presence of a nonappetitive odor.

In the experienced group, PER threshold to sucrose in MBablated flies significantly increased in the presence of the odor of D-limonene ( $p<0.05$, Steel-Dwass test; $n=50)$ but not in the absence of the odor (Fig. $3 B$ ). In PER duplicate tests, $\log _{10}\left[\mathrm{C}_{1 / 2}\right]$ was -0.81 in the absence of the odor and -0.36 in the presence of the odor (data from the curve in Fig. $3 B$ ) for one data set, and $\log _{10}\left[C_{1 / 2}\right]$ was -1.40 in the absence of the odor and -0.72 in the presence of the odor for another data set. This differed from results in the experienced group of intact flies (Fig. 3D). In PER duplicate tests, $\log _{10}\left[\mathrm{C}_{1 / 2}\right]$ was -0.38 in the absence of the odor and -0.30 in the presence of the odor (data from the curve in Fig. $3 D$ ) for one data set, and $\log _{10}\left[\mathrm{C}_{1 / 2}\right]$ was -0.65 in the absence of the odor and -0.40 in the presence of the odor for another data set. Regarding the absence or the presence of the odor of D-limonene, increase in PER threshold to sucrose in the experienced group of MB-ablated flies (Fig. 3B) was similar to that in the nonexperienced group of intact flies (Fig. 3C).

After behavioral experiments, 75 flies fed with HU-plus diet and 70 flies fed with HU-minus diet were randomly chosen and 


\section{HU-treated}

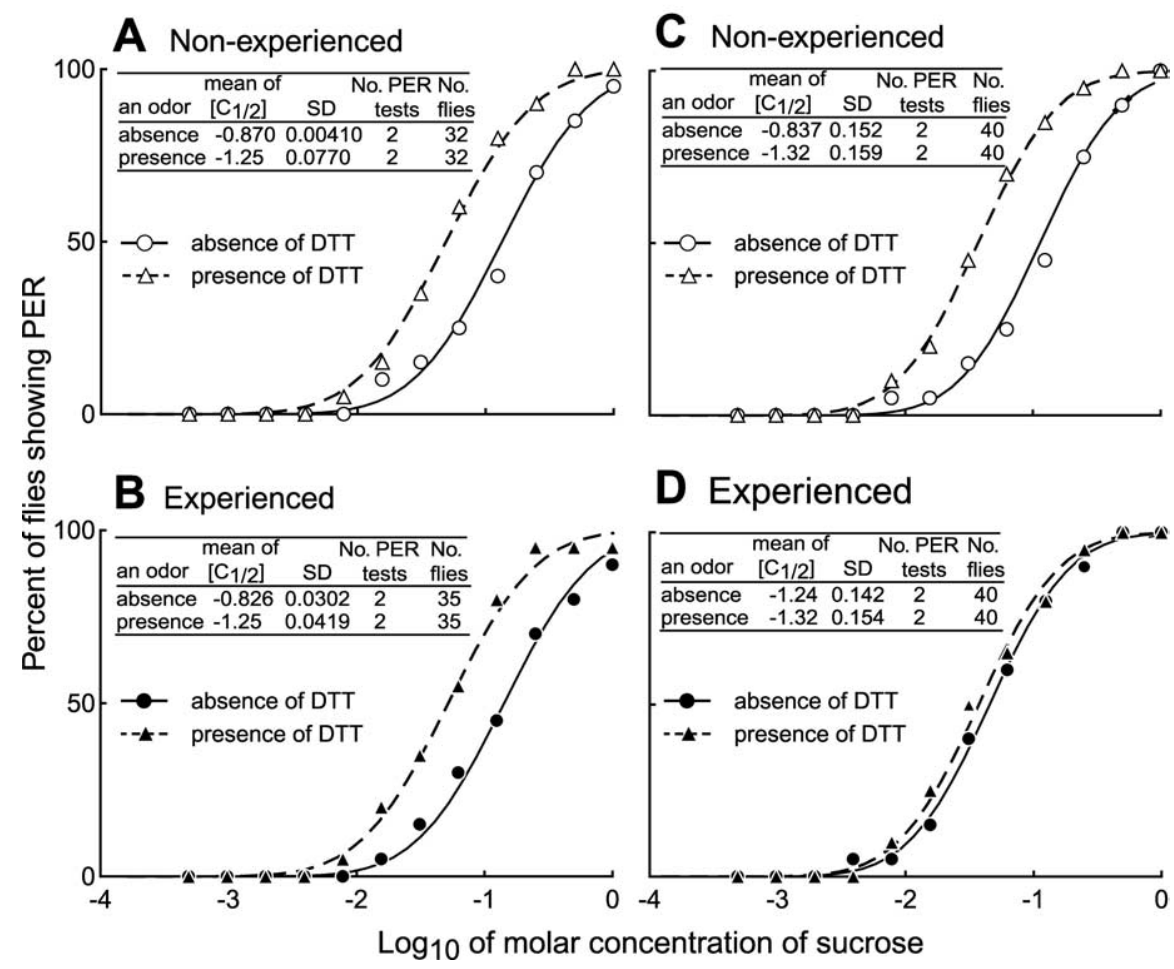

Figure 4. Effects of the odor of DTT on PER threshold to sucrose in MB-ablated flies. Newly hatched larvae were fed with HU-containing diet $(\boldsymbol{A}, \boldsymbol{B})$ or normal artificial diet $(\boldsymbol{C}, \boldsymbol{D})$. Adult flies were fed with $0.1 \mathrm{~m}$ sucrose without (open symbols; $A, C$ ) or with (filled symbols; $\boldsymbol{B}, \boldsymbol{D}$ ) the odor of DTT for $5 \mathrm{~d}$ after emergence. The PER test was performed $7 \mathrm{~d}$ after emergence in the absence (circles) or the presence (triangles) of the odor of DTT. Sigmoidal curves with circles or triangles show representative results of PER duplicate tests in a fly group.

subjected to histological examinations of their brain. Seventyfour brains of the 75 flies fed with HU-plus diet had no calyces, peduncles, or lobes (see Fig. 5, left). It was thus confirmed that MBs were completely ablated in these flies except for one specimen, in which MBs were entirely preserved. The larva might not eat sufficient amounts of the HU-plus diet or a contamination of blue stain of the diet on its outer body surface might have occurred. There were no cases in which MBs were partially ablated. All fly specimens fed with HU-minus diet preserved apparently intact MBs (see Fig. 5, right).

\section{Effects of the presence and dietary experience of the odor of DTT on PER threshold to sucrose in MB-ablated flies}

Using MB-ablated flies, we also investigated appetite modulation by an appetitive odor, DTT, and the effect of dietary experience. In PER duplicate tests using 12 and 20 flies from different batches, $\log _{10}\left[C_{1 / 2}\right]$ was -0.87 in the absence of the odor and -1.20 in the presence of the odor (data from the curve in Fig. $4 \mathrm{~A}$ ) for one data set, and $\log _{10}\left[\mathrm{C}_{1 / 2}\right]$ was -0.87 in the absence of the odor and -1.31 in the presence of the odor for another data set. In the nonexperienced group of MB-ablated flies, PER threshold to sucrose significantly decreased in the presence of DTT odor (Fig. $4 A)(p<0.05$, Steel-Dwass test; $n=32)$. This was similar to what was demonstrated in the nonexperienced group of intact flies (Fig. $4 C)(p<0.05$, Steel-Dwass test; $n=40)$. In PER duplicate tests using 20 and 20 flies from different batches, $\log _{10}\left[\mathrm{C}_{1 / 2}\right]$ was -0.73 in the absence of the odor and -1.20 in the presence of the odor (data from the curve in Fig. 4C) for one data set, and $\log _{10}\left[\mathrm{C}_{1 / 2}\right]$ was -0.94 in the absence of the odor and -1.43 in the presence of the odor for another data set. Even in MB-ablated flies, appetite could be modulated by an olfactory flavor, regardless of appetitive or nonappetitive odor. MBs probably were not needed to modulate appetite to sucrose in the presence of either nonappetitive or appetitive odor.

In the experienced group, PER duplicate tests using 15 and 20 flies from different batches revealed that $\log _{10}\left[\mathrm{C}_{1 / 2}\right]$ was -0.80 in the absence of the odor and -1.22 in the presence of the odor (data from the curve in Fig. $4 B$ ) for one data set, and $\log _{10}\left[\mathrm{C}_{1 / 2}\right]$ was -0.85 in the absence of the odor and -1.28 in the presence of the odor for another data set. PER threshold to sucrose in MB-ablated flies significantly decreased in the presence of DTT odor (Fig. $4 B)(p<0.05$, Steel-Dwass test; $n=35$ ). This was similar to results in the nonexperienced group of intact flies (Fig. 4C) but different from results in the experienced group of intact flies (Fig. $4 D$ ). In PER duplicate tests using 20 and 20 flies from different batches, $\log _{10}\left[\mathrm{C}_{1 / 2}\right]$ was -1.14 in the absence of the odor and -1.21 in the presence of the odor (data from the curve in Fig. $4 D$ ) for one data set, and $\log _{10}\left[\mathrm{C}_{1 / 2}\right]$ was -1.34 in the absence of the odor and -1.43 in the presence of the odor for another data set. In terms of absence or presence of DTT odor, decrease in PER threshold to sucrose in the experienced group of MB-ablated flies (Fig. $4 B$ ) was similar to that in the nonexperienced group of intact flies (Fig. 4C).

After the behavioral experiments, of 52 flies fed with HU-plus diet, 39 flies were randomly chosen for histological examinations of their brain, and, of 80 flies fed with HU-minus diet, 21 flies were randomly chosen for histological examinations of their brain. All brains from flies fed with HU-plus diet showed completely ablated MBs (Fig. 5, left). There were no cases in which MBs were partially ablated. All brains from flies fed with HUminus diet showed completely preserved MBs (Fig. 5, right).

\section{Changes in biogenic amine levels in intact brains between experienced and nonexperienced flies}

In our HPLC-ECD protocol, six kinds of biogenic amines, OA, NADA, DA, NATA, TA, and 5-HT, could be detected simultaneously. Brain extracts of flies, which had a dietary experience with the odor of D-limonene, contained significantly lower amounts of $\mathrm{OA}$ and TA than those of flies that had no dietary experience with the odor (Fig. $6 A)(p<0.05$, Mann-Whitney $U$ test; $n=15$ ).

In contrast, brain extracts of flies that had a dietary experience with DTT odor $(n=18)$ contained significantly higher amounts of OA and TA than those of flies that had no dietary experience with the odor $(n=16)$ (Fig. $6 B)(p<0.05$, Mann-Whitney $U$ test). Other amines showed no significant difference between the experienced $(n=18)$ and the nonexperienced $(n=16)$ flies (Fig. $6 B)(p>0.05$, Mann-Whitney $U$ test). These results suggested that OA and/or TA were involved in appetite level, which could 
be modulated by dietary experience with either a nonappetitive or an appetitive odor.

Changes in biogenic amine levels in MBablated brains between experienced and nonexperienced flies

MB-ablated flies, even when given a dietary experience with a nonappetitive or an appetitive odor after emergence, did not exhibit a significant appetite change to sucrose (Figs. 3, 4). Thus, it is questionable whether MB-ablated flies that once had a dietary experience with a nonappetitive or an appetitive odor show any changes in $\mathrm{OA}$ and/or TA levels in the brain, although appetite change was not induced.

Figure 7 shows that, in MB-ablated flies, even when given a dietary experience with the odor of D-limonene or DTT, there is no significant decrease in either OA or TA levels in brain $(p>0.05$, Mann-Whitney $U$ test; $n=10-14$ ).

\section{Effects of injections of OA/TA receptor agonists and antagonists on PER threshold to sucrose}

We observed a parallel relationship between OA and TA levels in brain with flies' appetite, which was measured by PER. When Ringer's solution was injected, PER threshold to sucrose still increased after dietary experience with the odor of D-limonene. The values of $\log _{10}\left[\mathrm{C}_{1 / 2}\right]$ obtained by the PER test in flies that had no dietary experience with the odor of D-limonene was $-1.30 \pm 0.282(n=5)$ and that in flies that had a dietary experience with the odor was $-0.847 \pm 0.281$ $(n=5)$ (Fig. $8 A$ ). When $0.2 \mu \mathrm{g}$ of OA dissolved in $1 \mu$ l of Ringer's solution was injected, $\log _{10}\left[\mathrm{C}_{1 / 2}\right]$ was $-0.924 \pm 0.254(n=8)$. PER threshold, which was statistically tested at the individual level $(n=208)$, hardly changed from that when plain Ringer's solution was injected $(n=120)$ (Fig. $8 A)(p>0.05$, Steel-Dwass test).

However, when $0.2 \mu \mathrm{g}$ of TA dissolved in $1 \mu \mathrm{l}$ of Ringer's solution was injected, $\log _{10}\left[\mathrm{C}_{1 / 2}\right]$ was $-1.23 \pm 0.459(n=12)$ (Fig. $8 \mathrm{~B}$ ). This was lower than the average value of the mean PER threshold in flies with no dietary experience with the odor, $-0.964 \pm 0.247(n=8)$. PER threshold at the individual level $(n=290)$ significantly decreased from that when Ringer's solution was injected $(n=170)(p<0.05$, Steel-Dwass test). This indicated that TA injection rather than OA injection effectively increased appetite that was measured by PER to sucrose in the blowfly. When clonidine hydrochloride, an agonist to OA/TA receptor, was injected, the results mostly agreed with those obtained with TA injection (Fig. 8C). Moreover, we demonstrated a TA dose dependency of the decrease in PER threshold in Figure $8 D$. The optimum concentration of TA was $\sim 0.4 \mu \mathrm{g} / \mu \mathrm{l}$.

We could also experimentally set an increased appetite in flies by providing a dietary experience with DTT odor. To such flies, we injected phentolamine, an OA/TA receptor inhibitor, and examined its effects on appetite definition in flies (Fig. 9). When 1 $\mu \mathrm{l}$ of plain Ringer's solution was injected to flies that had no dietary experience with the odor, $\log _{10}\left[\mathrm{C}_{1 / 2}\right]$ was $-0.876 \pm$ $0.0875(n=2)$. When flies had a dietary experience with DTT odor, $\log _{10}\left[\mathrm{C}_{1 / 2}\right]$ decreased to $-1.31 \pm 0.133(n=2)(p<0.05$, at individual level by Steel-Dwass test; $n=40)$. When $0.2 \mu \mathrm{g}$ of phentolamine dissolved in $1 \mu \mathrm{l}$ of Ringer's solution was injected to such experienced flies, PER threshold significantly increased to $-0.639 \pm 0.0557(n=2)(p<0.05$, at individual level by SteelDwass test; $n=40)$. This value was similar to that when $0.2 \mu \mathrm{g}$ of phentolamine was injected to nonexperienced flies, i.e., $-0.621 \pm 0.0373(n=2)(p>0.05$, at individual level by SteelDwass test; $n=40)$.

Throughout the injection experiments, we suggested that some TA-TA receptor synapses probably in MBs were involved in appetite definition, which was influenced by previous dietary experiences with odors.

\section{Discussion}

\section{Olfactory influence on appetite and feeding behavior}

in insects

There are many psychological studies on olfactory effects on appetite, feeding preference, or other emotional changes in humans. However, there are few reports in insects, suggesting that appetite, behaviorally evaluated by PER threshold to a taste stimulus, is modulated in the presence of odors. The present study 

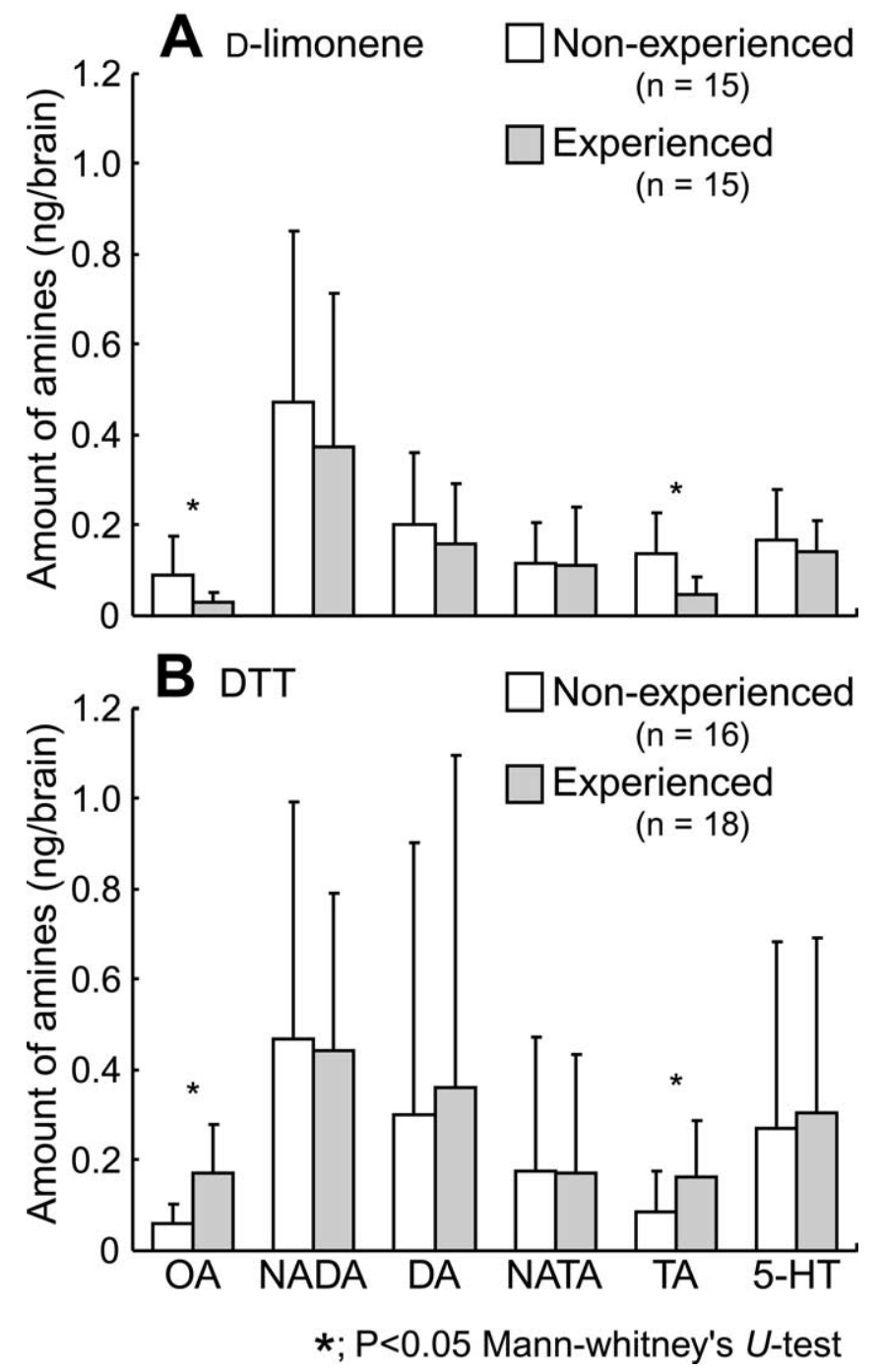

Figure 6. Changes in biogenic amine levels in brains of experienced and nonexperienced flies. Amounts of measured biogenic amines are indicated in brains of flies, which were fed with $0.1 \mathrm{~m}$ sucrose without (open columns) or with (filled columns) the odor of $\mathrm{D}$-limonene $(\boldsymbol{A})$ or DTT $(\boldsymbol{B})$ for $5 \mathrm{~d}$ after emergence. The asterisk indicates a significant difference in the amounts of measured biogenic amines between nonexperienced (open columns) and experienced fly groups (filled columns). Error bars indicate SDs.

using PER test showed that the odors of D-limonene and DTT are nonappetitive or appetitive for the blowfly $P$. regina, respectively (Figs. 1, 2).

The ecological significance of the effect of DTT odor is unclear. However, $P$. regina adults are known to visit flowers to feed on nectar (Nye and Anderson, 1974; Larson et al., 2001). Flies are exposed to odors of various components of floral scents when visiting flowers in the field. D-Limonene is not only a component of some floral scents (Knudsen et al., 1993) but also a secondary metabolite of plants, which generally acts as a repellent for different groups of phytophagous insects. Indeed, D-limonene is a dangerous substance because of its oral toxicity in a wide range of insect species (Taylor and Vickery, 1974; Hink and Fee, 1986; Ozaki et al., 2003; Tripathi et al., 2003). Thus, increase in PER thresholds by the odor of D-limonene would be beneficial for flies in preventing them from taking foods containing D-limonene before tasting them (Ozaki et al., 2003). Flies may have constructed neural systems to associate olfactory cues of oral toxins
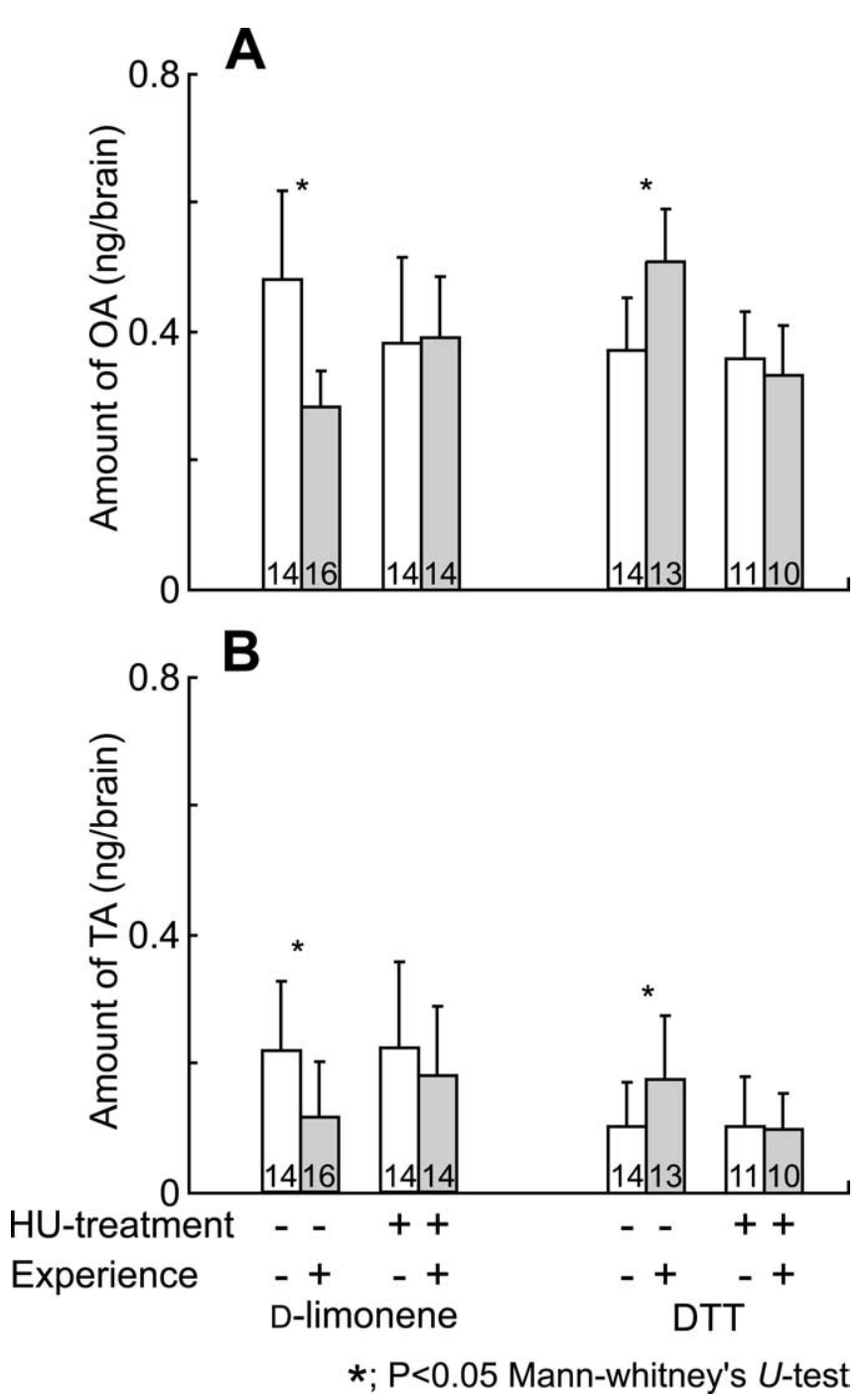

Figure 7. Effects of MB ablation on changes in $0 \mathrm{~A}$ and TA levels in brains of experienced and nonexperienced flies. Newly hatched larvae were fed with $\mathrm{HU}$-containing diet or normal artificial diet. Amounts of $0 A(\boldsymbol{A})$ and TA $(\boldsymbol{B})$ are indicated in brains of flies, which were fed with 0.1 m sucrose without (open columns) or with (filled columns) the odor of D-limonene or DTT for $5 \mathrm{~d}$ after emergence. The asterisk indicates a significant difference in the amounts of $0 \mathrm{~A}$ and TA between nonexperienced and experienced fly groups. Error bars indicate SDs. Numbers indicate the number of samples.

such as D-limonene with gustatory inputs of foods, resulting in behavioral output for a safe food choice for survival.

The present study also showed that dietary experiences with odors of D-limonene and DTT increased and decreased PER threshold, respectively (Figs. 1, 2). PER threshold, which may be differentially affected by environmental factors and previous experiences, can affect behavioral choices for feeding. It is most probable that flies would integrate olfactory cues with gustatory cues to tune PER thresholds, so that they can choose the best behavioral output based on the most likely cost and benefit balance in every feeding attempt.

Involvement of $\mathrm{MB}$ in appetite modulation by dietary experiences with nonappetitive or appetitive odors

In intact flies, PER thresholds were modulated by previous feeding experiences with nonappetitive or appetitive odors, mimicking the presence of olfactory stimuli (Figs. 1, 2). Naturally, the taste of sucrose used here sends out a strong signal of an energy 
food source. Odors of D-limonene and DTT are not biologically neutral but are innately nonappetitive and appetitive in this species, respectively. During the experience period, flies may somehow relate the odors of D-limonene or DTT with the taste of sucrose. As a result, it was presumed that PER threshold to sucrose decreased or increased, even in the absence of the odor of D-limonene or DTT, respectively.

On the contrary, in MB-ablated flies, PER thresholds would not be modulated by previous feeding experiences with nonappetitive or appetitive odors (Figs. 3, 4). They appeared to ignore dietary experiences.

de Belle and Heisenberg (1994) demonstrated that MBs of D. melanogaster were ablated by treating first-instar larvae with the cytostatic drug HU. Then, the four MB neuroblasts were ablated in each brain hemisphere that generated all postembryonic Kenyon cells (Prokop and Technau, 1994), and the fifth neuroblast contributed neurons to the formation of the antennal lobe (AL). Although it was reported in $D$. melanogaster that $\mathrm{MBs}$ were partially ablated in $6.5 \%$ of HU-treated flies (de Belle and Heisenberg, 1994), such a partial ablation was shown in D. melanogaster and was not found in our study with P. regina (Fig. 5) and Protophormia terraenovae (Ikeda et al., 2005). There might be some differences in time course and temporal patterns of cell division of neuroblasts between $D$. melanogaster and $P$. regina or $P$. terraenovae. In $P$. terraenovae, reduction in the volume of ALs was detected (Ikeda et al., 2005). It may also occur in $P$. regina, although we did not investigate this matter in details as to have a final conclusion on AL reduction.

In our study, it was important to determine whether MBablated flies could smell D-limonene or DTT. We did not directly show olfactory responses of antennae to these odors in intact and MB-ablated flies after HU treatment. However, our data revealed that not only intact but also MB-ablated flies showed similar PER modulations in the presence of odors of D-limonene or DTT (Figs. 3, 4). This suggested that, even without MBs, flies could integrate food information from taste and olfactory cues, which were given to flies at the same time, to regulate their appetite. MB-ablated flies could smell either D-limonene or DTT.

Conversely, MB has been repeatedly documented as a particularly important central neuropil concerned with memory. It is indispensable in insect brains for associative learning of olfactory information (Heisenberg, 1998; Menzel, 2001; Roman and Davis, 2001). In D. melanogaster, associative learning has been extensively studied using neutral odors as conditioned stimuli and electric shock as an aversive unconditioned stimulus (Tully and Quinn, 1985; Dubnau and Tully, 1998; Waddell and Quinn, 2001; Schwaerzel et al., 2003). It was reported that associative learning between odor and electric shock was abolished by chem- ical ablation of MBs (de Belle and Heisenberg, 1994) and by perturbations in cAMP signaling within MBs (Connolly et al., 1996). Nevertheless, simple odor detection was not affected. Recently, McGuire et al. (2001) and Dubnau et al. (2001) showed that synaptic output from MBs was required for recalling olfactory memory but not for its acquisition or storage.

In the present study, it seemed that memory of previous dietary experience with nonappetitive or appetitive odor was recalled when flies were given sucrose taste stimulation (Figs. 1, 2). We did not perform experimental conditioning for traditional associative learning. Therefore, we cannot conclude that some sorts of associative learning are involved in appetite modulation by dietary experience with a nonappetitive or an appetitive odor. However, we can claim that $P$. regina could integrate food information from sugar taste and olfactory flavors, and that appetite modulations based on dietary experiences would occur through $\mathrm{MB}$ as a memory center. Not only in flies but also in other animals, individual food preference might be formed, being based on two basic capabilities: one involves recognizing palatability by 


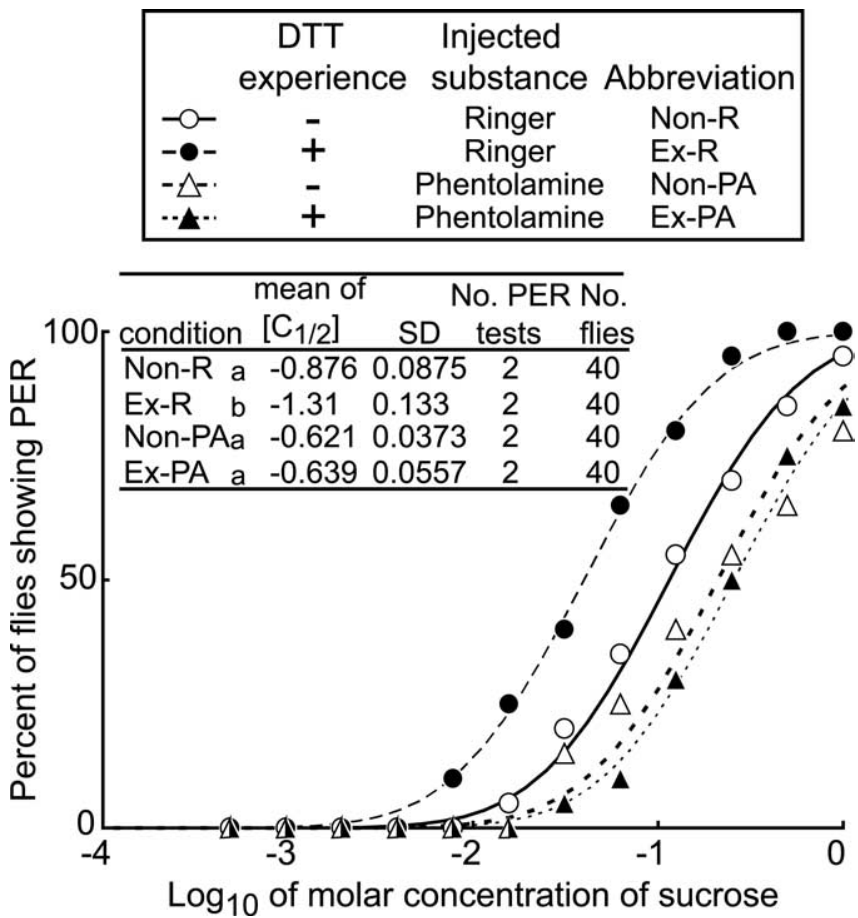

Figure 9. Effects of injections of phentolamine on PER threshold to sucrose that decreased by dietary experience with the odor of DTT. Flies were fed with $0.1 \mathrm{~m}$ sucrose without or with the odor of DTT for $5 \mathrm{~d}$ after emergence. Plain or phentolamine-containing Ringer's solution was injected into experienced (filled symbols) and nonexperienced flies (open symbols). Sigmoidal curves with circles and triangles show representative results of PER duplicate tests in a fly group. Conditions having the same alphabetical letter in each table indicate nonsignificant differences ( $p>0.05$, Steel-Dwass test). Non, Nonexperienced; Ex, experienced; R, Ringer's solution; PA, phentolamine.

integration of taste and olfactory food flavors, and the other involves memorizing dietary experiences.

\section{Putative role of TA for appetite regulation}

In insects, OA is known as a neurotransmitter and neuromodulator, but the physiological role of TA remains unclear (Roeder et al., 2003). TA has been known as a precursor of OA but has not been thought to have any physiological functions of its own. Recently, it was reported in D. melanogaster that TA acts as a modulatory transmitter at the larval neuromuscular junction (Kutsukake et al., 2000). In honeybee, PER thresholds to sucrose decreased by injection and prescription of TA or OA (Scheiner et al., 2002). In the blowfly, it was reported previously that injection of OA $(75 \mu \mathrm{g} / \mathrm{fly}$ ) decreased PER thresholds (Long and Murdock, 1983). In our case, injection of OA (0.2-2.0 $\mu \mathrm{g} / \mathrm{fly})$ hardly changed the PER threshold (Fig. 8). This discrepancy was probably attributable to the differences in the injected amounts of OA.

We showed that injection of TA decreased PER threshold with an optimum concentration of $\sim 0.4 \mu \mathrm{g} / \mu \mathrm{l}$ (Fig. 8 ). Nevertheless, it was still possible to explain that injected TA did not directly but indirectly affected PER, because OA produced from TA could affect PER. As mentioned previously, injection of OA had no effect on PER (Fig. 8); therefore, it is strongly suggested that not OA but TA directly affects $P E R$ in $P$. regina.

Biogenic amines have also been suggested to play an important role in associative learning. In honeybee, the ventral unpaired medial neuron, depolarization of which substitutes for sucrose stimulation in the conditioning of PER (Hammer, 1993), is an octopaminergic cell (Kreissl et al., 1994). When OA was injected into the MB calyces as a substitute for sucrose stimulation, paired with the odor, a pairing-specific enhancement of PER occurred (Hammer and Menzel, 1998). In D. melanogaster, when an odor was conditioned with sucrose or electric shock, different biogenic amines were involved in memory formation: DA in appetitive conditioning and OA in aversive conditioning (Schwaerzel et al., 2003). However, there were few reports suggesting an importance of TA in olfactory associative learning.

In our experiments, depending on the previously experienced odor, the amount of TA in the intact fly brain was altered (Fig. 6). In the MB-ablated fly, however, the amount of TA in the brain was unchanged by dietary experience (Fig. 7). These results suggested that change in appetite caused by dietary experiences with nonappetitive or appetitive odors was mediated by changes of TA levels in the brain, and target points of TA in the neural system might be related to MBs. To confirm this, additional physiological and morphological investigations in the brains of the flies, with different appetite levels, are needed.

\section{References}

Akahane R, Amakawa T (1983) Stable and unstable phase of memory in classically conditioned fly, Phormia regina: effects of nitrogen gas anaesthesia and cycloheximide injection. J Insect Physiol 29:331-337.

Ayyub C, Paranjape J, Rodrigues V, Siddiqi O (1990) Genetics of olfactory behavior in Drosophila melanogaster. J Neurogenet 6:243-262.

Blenau E, Erber J (1998) Behavioural pharmacology of dopamine, serotonin and putative aminergic ligands in the mushroom bodies of the honeybee (Apis mellifera). Behav Brain Res 96:115-124.

Brigui N, Le Bourg E, Médioni J (1990) Conditioned suppression of the proboscis-extension response in young, middle-aged, and old Drosophila melanogaster flies: acquisition and extinction. J Comp Psychol 104:289-296.

Brookhart GL, Edgecomb RS, Murdock LL (1987) Amphetamine and reserpine delete brain biogenic amines and alter blow fly feeding behavior. J Neurochem 48:1307-1315.

Connolly JB, Roberts IJ, Armstrong JD, Kaiser K, Forte M, Tully T, O’Kane CJ (1996) Associative learning disrupted by impaired Gs signaling in Drosophila mushroom bodies. Science 274:2104-2107.

de Belle JS, Heisenberg M (1994) Associative odor learning in Drosophila abolished by chemical ablation of mushroom bodies. Science 263:692-695.

DeJianne D, McGuire TR, Pruzan-Hotchkiss A (1985) Conditioned suppression of proboscis extension in Drosophila melanogaster. J Comp Psychol 99:74-80.

Dethier VG (1976) The hungry fly. Cambridge, MA: Harvard UP.

Devaud J-M (2003) Experimental studies of adult Drosophila chemosensory behaviour. Behav Processes 64:177-196.

Dubnau J, Tully T (1998) Gene discovery in Drosophila: new insights for learning and memory. Annu Rev Neurosci 21:407-444.

Dubnau J, Grady L, Kitamoto T, Tully T (2001) Disruption of neurotransmission in Drosophila mushroom body blocks retrieval but not acquisition of memory. Nature 411:476-480.

Erber J, Kloppenburg P, Scheidler A (1993) Neuromodulation by serotonin and octopamine in the honeybee: behavior, neuro-anatomy and electrophysiology. Experientia 49:1073-1083.

Evans PD (1980) Biogenic amines in the insect nervous system. Adv Insect Physiol 15:317-473.

Hammer M (1993) An identified neuron mediates the unconditioned stimulus in associative olfactory learning in honeybees. Nature 366:59-63.

Hammer M, Menzel R (1998) Multiple sites of associative odor learning as revealed by local brain microinjections of octopamine in honeybees. Learn Mem 5:146-156.

Heisenberg M (1998) What do the mushroom bodies do for the insect brain? An introduction. Learn Mem 5:1-10.

Hink WF, Fee BJ (1986) Toxicity of D-limonene, the major component of citrus peel oil, to all life stages of the cat flea, Ctenocephalides felis (Siphonaptera: Pulicidae). J Med Entomol 23:400-404.

Ikeda K, Numata H, Shiga S (2005) Roles of the mushroom bodies in olfactory learning and photoperiodism in the blowfly Protophormia terraenovae. J Insect Physiol 51:669-680. 
Ishimoto H, Tanimura T (2004) Molecular neurophysiology of taste in Drosophila. Cell Mol Life Sci 61:10-18.

Knudsen JT, Tollsten L, Bergstrom LG (1993) Floral scents-a checklist of volatile compounds isolated by head space techniques. Phytochemistry 33:253-280.

Kreissl S, Eichmüller G, Bicker J, Rapus J, Eckert M (1994) Octopamine-like immunoreactivity in the brain and suboesophageal ganglion of the honeybee. J Comp Neurol 348:583-594.

Kutsukake M, Komatsu A, Yamamoto D, Ishiwa-Chigusa S (2000) A tyramine receptor gene mutation causes a defective olfactory behavior in Drosophila melanogaster. Gene 245:31-42.

Larson BMH, Kevan PG, Inouye DW (2001) Flies and flowers: taxonomic diversity of anthophilesand pollinators. Can Entomol 133:439-465.

Long TF, Murdock LL (1983) Stimulation of blowfly feeding behavior by octopaminergic drugs. Proc Natl Acad Sci USA 80:4159-4163.

Long TF, Edgecomb RS, Murdock LL (1986) Effects of substituted phenylethylamines on blowfly feeding behavior. Comp Biochem Physiol C 83:201-209.

McGuire SE, Le PT, Davis RL (2001) The role of Drosophila mushroom body signaling in olfactory memory. Science 293:1330-1333.

Médioni J, Vaysse G (1975) Suppression conditionelle d'un réflexe chez la Drosophile (Drosophila melanogaster): acquisition et extinction. CR Seances Soc Biol Fil 169:1386-1391.

Menzel R (2001) Searching for the memory trace in a mini-brain, the honeybee. Learn Mem 8:53-62.

Nagao T, Tanimura T (1988) Distribution of biogenic amines in the cricket central nervous system. Anal Biochem 171:33-40.

Nagao T, Tanimura T (1989) Simultaneous determination of biogenic amines, their precursors and metabolites in a single brain of the cricket using high-performance liquid chromatography with ameperometric detection. J Chromatogr 496:39-53.

Nye WP, Anderson JL (1974) Insect pollinators frequenting strawberry blossoms and the effect of honey bees on yield and fruit quality. J Am Soc Hort Sci 99:40-44.

Ozaki M, Takahara T, Kawahara Y, Wada-Katsumata A, Seno K, Amakawa T, Yamaoka R, Nakamura T (2003) Perception of noxious compounds by contact chemoreceptors of the blowfly, Phormia regina: putative role an odorant-biding protein. Chem Senses 28:349-359.

Page Jr RE, Erber J (2002) Levels of behavioral organization and the evolution of division of labor. Naturwissenschaften 89:91-106.

Pankiw T, Page Jr RE (1999) The effect of genotype, age, sex, and caste on response thresholds to sucrose and foraging behavior of honey bees (Apis mellifera L.) J Comp Physiol A Neuroethol Sens Neural Behav Physiol 185:207-213.

Pankiw T, Waddington KD, Page Jr RE (2001) Modulation of sucrose response thresholds in honey bees (Apis mellifera L.): influence of genotype, feeding, and foraging experience. J Comp Physiol A Neuroethol Sens Neural Behav Physiol 187:293-301.

Prokop A, Technau GM (1994) Normal function of the mushroom body defect gene of Drosophila is required for the regulation of the number and proliferation of neuroblasts. Dev Biol 161:321-337.

Roeder T (1994) Biogenic amines and their receptors in insects. Comp Biochem Physiol C Pharmacol Toxicol Endocrinol 107:1-12.

Roeder T, Seifert M, Kähler C, Gewecke M (2003) Tyramine and octopamine: agonistic modulators of behavior and metabolism. Arch Insect Biochem Physiol 54:1-13.

Roman G, Davis LR (2001) Molecular biology and anatomy of Drosophila olfactory associative learning. BioEssays 23:571-581.

Sakuma M (1998) Probit analysis of preference data. Appl Entomol Zool 33:339-347.

Scheiner R, Pluckhahn S, Oney B, Blenau W, Erber J (2002) Behavioural pharmacology of octopamine, tyramine and dopamine in honey bees. Behav Brain Res 136:545-553.

Scheiner R, Page RE, Erber J (2004) Sucrose responsiveness and behavioral plasticity in honey bees (Apis mellifera). Aidologie 35:133-142.

Schwaerzel M, Monastirioti M, Scholz H, Friggi-Grelin F, Birman S, Heisenberg M (2003) Dopamine and octopamine differentiate between aversive and appetitive olfactory memories in Drosophila. J Neurosci 23:10495-10502.

Tachibana S-I, Numata H (2001) An artificial diet for blow fly larvae, Lucilia sericata (Meigen) (Diptera: Calliphoridae). Appl Entomol Zool 36:521-523.

Taylor WE, Vickery B (1974) Insecticidal properties of limonene, a constituent of citrus oil. Ghana J Agri Sci 7:61-62.

Tripathi AK, Prajapati V, Khanuja SP, Kumar S (2003) Effect of d-limonene on three stored-product beetles. J Econ Entomol 96:990-995.

Tully T, Quinn WG (1985) Classical conditioning and retention in normal and mutant Drosophila melanogaster. J Comp Physiol A Neuroethol Sens Neural Behav Physiol 157:263-277.

Waddell S, Quinn WG (2001) Flies, genes, and learning. Annu Rev Neurosci 24:1283-1309.

Yano T, Nakashima M, Shiraishi A, Morita H (1986) Relationship between elevation of the labellar threshold after raising be increasing sucrose concentrations and impulse of a single sugar receptor in the LL-type hair of the blowfly. Exp Biol 46:29-35. 